\title{
Progress and future prospect of the CRAFFT project for the next generation UHECR observation
}

\author{
Yuichiro Tameda, ${ }^{a, *}$ Takayuki Tomida, ${ }^{b}$ Daisuke Ikeda, ${ }^{c}$ Katsuya Yamazaki, ${ }^{d}$ Takashi \\ Kagitani, ${ }^{a}$ Norimichi Shibata, ${ }^{a}$ Eiji Nishio, ${ }^{a}$ Hirokazu Iwakura, ${ }^{b}$ Yuya Nakamura, ${ }^{b}$ \\ Yuto Kubota, ${ }^{b}$ Shinnosuke Kurisu ${ }^{b}$ and Ryosuke Saito ${ }^{b}$ \\ ${ }^{a}$ Osaka Electro-Communication University, Department of Engineering Science, Neyagawa, Osaka, Japan \\ ${ }^{b}$ Shinshu University, Faculty of Engineering, Nagano, Nagano, Japan \\ ${ }^{c}$ Kanagawa University, Faculty of Engineering, Yokohama, Kanagawa, Japan \\ ${ }^{d}$ Chubu university, Engineering Science Laboratory, Kasugai, Aichi, Japan \\ E-mail: tameda@osakac.ac.jp
}

\begin{abstract}
Signs of anisotropy of the ultra-high-energy cosmic ray (UHECR) arrival direction have been reported by The Telescope Array and The Pierre Auger Observatory. Then, it is expected that source of UHECRs will be clarified. However, because of the quite low flux of UHECR, it is inevitable to build a much larger observatory than the existing one. Cosmic Ray Fluorescence Fresnel lens Telescope (CRAFFT) project has been developed a low cost fluorescence detector to realize a huge observatory for UHECR observation. CRAFFT project reduced the cost to $1 / 10$ against fluorescence detectors now in use, and succeeded to observe UHECR air showers by the prototype of CRAFFT telescope. In this presentation, we will report the progress of CRAFFT project such as the result of the test observation and data analysis and discuss the future prospect.
\end{abstract}

$37^{\text {th }}$ International Cosmic Ray Conference (ICRC 2021)

July 12th-23rd, 2021

Online - Berlin, Germany

\footnotetext{
${ }^{*}$ Presenter
} 


\section{Introduction}

Recently, there are many results about the arrival direction analysis from Pierre Auger Observatory (Auger) and Telescope Array (TA). For example, these is a Hotspot in the arrival direction of ultra high energy cosmic rays (UHECRs) with energies above $57 \mathrm{EeV}$ reported by TA [1]. If the hotspot is related to the population of UHECR sources, what should we do to identify the UHECR sources? We need large statistics, but the flux of UHECR is quite low. Then, we need to build a much larger observatory than the existing one to earn large statistics.

It is also important to know the mass composition of UHECRs for the identification of their sources. Whether cosmic rays can propagate straight or not depends on primary energy and particle type. Then, we have to observe $X_{\max }$ or other sensitive parameter to primary particle type.

One of the solution is a huge observatory using fluorescence detector (FD) which can observe $X_{\max }$. However the cost of conventional FDs like TA or Auger is high. Then, we need to develop low cost FDs such as P. Privitera had proposed before[2].

\section{Instrument}

We are developing a low cost FD. It is Cosmic ray Fluorescence Fresnel lens Telescope named as CRAFFT. The figure 1 is the exterior of CRAFFT detector which has deployed at TA site. CRAFFT consists of a $1.4 \mathrm{~m}^{2}$ Fresnel lens, an 8 inc. PMT, a UV transmitting filter, and an FADC board. The cost of CRAFFT is about one tenth of conventional FD. The features of CRAFFT are simple structure, without container, easy to deploy, and no obstacle between lens and focus.

CRAFFT has only single pixel so that it is not easy to reconstruct shower geometry by monocular analysis. Then, stereo or multiple observation will be necessary. And, signal noise ratio also will be worse compared to conventional FDs.

Then, we have to demonstrate air shower detection and possibility of air shower reconstruction.
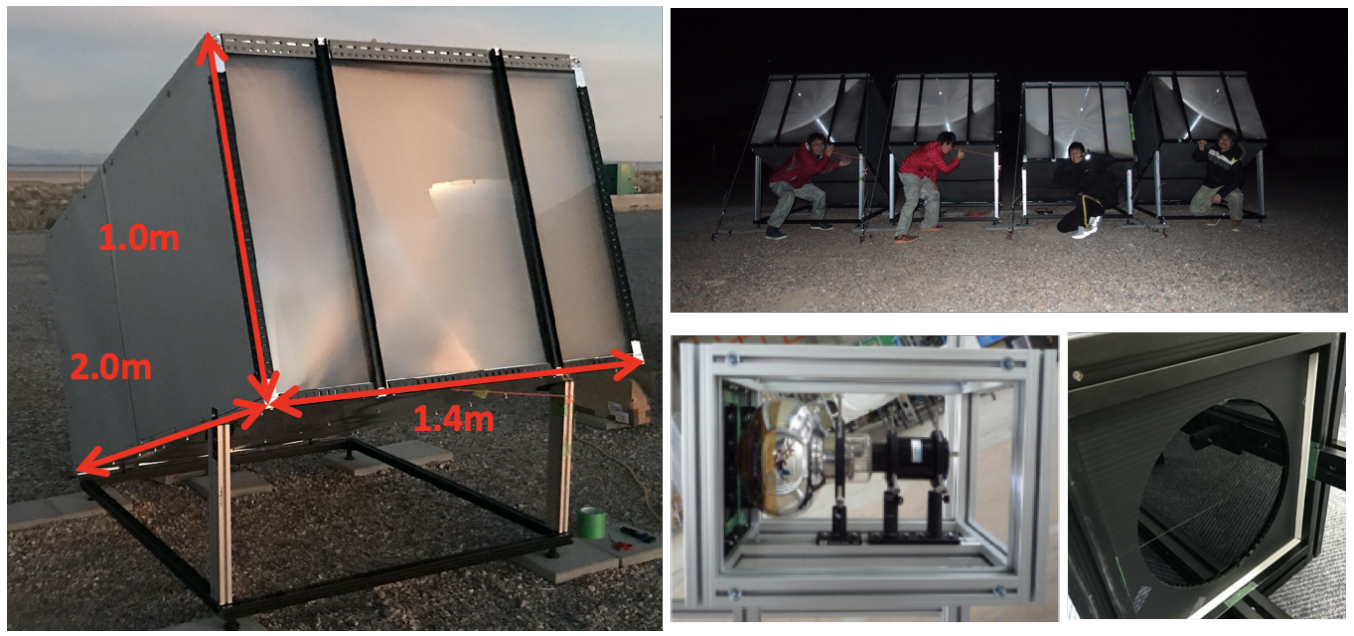

Figure 1: The exterior of the CRAFFT telescope (Left). Four telescopes was deployed at the Telescope Array Black Rock Mesa site (Upper right). 8 inc. PMT with UV transmitting filter is mounted at the focus point of the Fresnel lens and spatial filter is attached to the UV transmitting filter to adjust the field of view. 


\section{Test observation}

We have deployed four CRAFFT telescopes at the TA Black Rock Mesa site as shown in the Fig. 2. The CRAFFT telescope field of view coincides with the TA FD in the same direction as the central laser facility in the upper field of view. The expected number of events with the CRAFFT telescope was estimated to be about 8 events per month at energies above $10^{17} \mathrm{eV}$. Test observation period was from November 9 to 23, 2017 [3]. The observation time was 10 nights (63.5 hours). In the test observation, the data was acquired by using the external trigger from TA FD. The number of events recorded by the trigger of TA FD was 556,255. From these data, we selected events that were clearly air showers. At least 10 events were determined to be air shower events.

The figure 3 shows examples of air shower events detected by the CRAFFT telescope. In the case of this event, two of the four detectors have a clear signal. Comparing this event with the corresponding TA FD event display, we found that the air shower track goes through the field of view of CRAFFT telescopes which have signal. According to the TA FD analysis results, the primary energy is $10^{17.7} \mathrm{eV}$, and the core position was found to be $3.6 \mathrm{~km}$ from the detector.

Test observations have demonstrated the ability of the CRAFFT detector to detect cosmic rays.
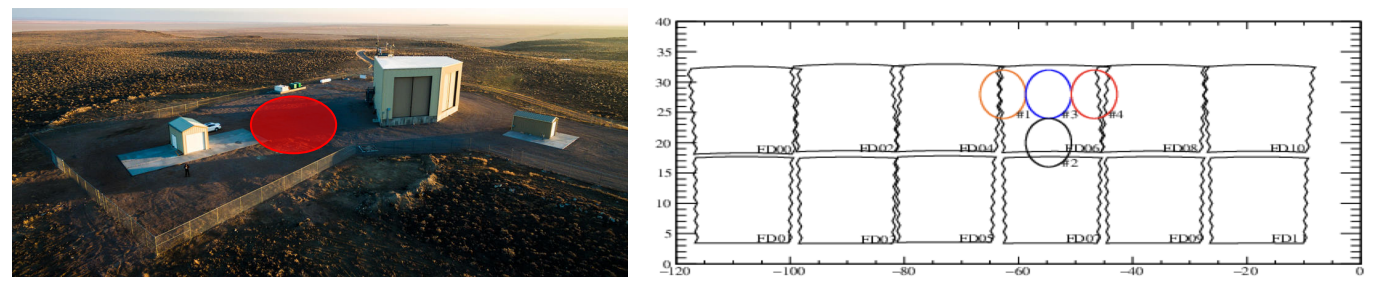

Figure 2: Left: CRAFFT is deployed between the TA FD building and FAST detector at Black Rock Mesa site. Right: Field of view of CRAFFT telescopes compared with TA FD field of view.
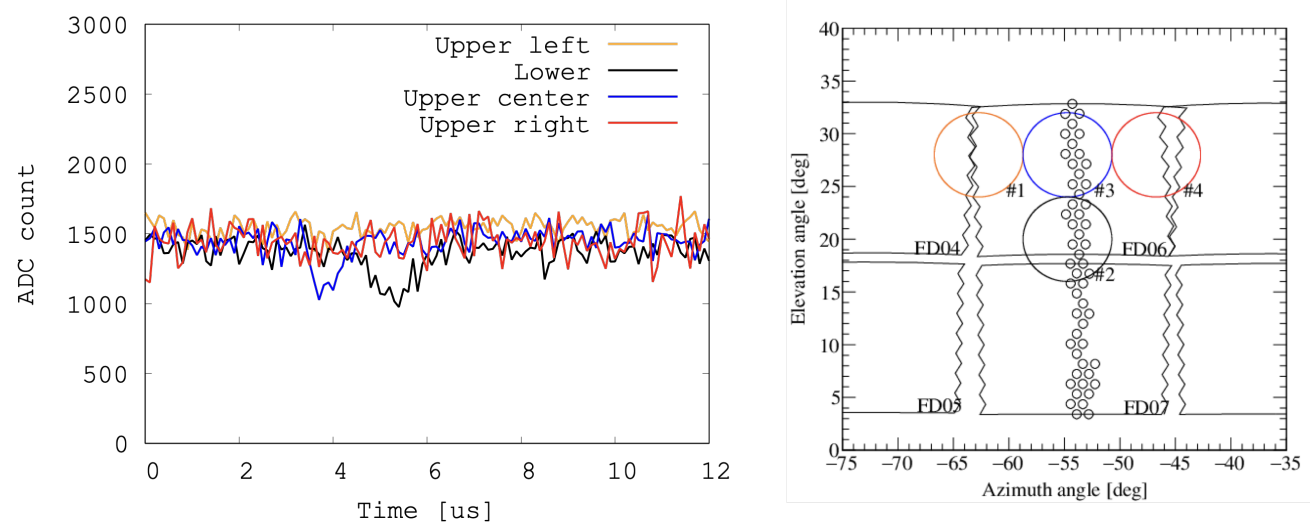

Figure 3: Typical air shower event acquired by CRAFFT. Left: The FADC count of each channel. Right: TA FD event display. The solid line is the edge of the TA FD FOV. The $1^{\circ}$ circles are views of the TA FD triggered channels. The $8^{\circ}$ circles are the FOV of CRAFFT. 


\section{Analysis framework}

The CRAFFT telescope has a different detector configuration from conventional FDs. A new analytical framework is required to estimate the detector performance or to reconstruct air showers. The CRAFFT has a wider field of view per pixel, which results in a worse signal-to-noise ratio compared to conventional FD. In order to determine the arrangement of detector array, the trigger efficiency must be carefully estimated by simulation.

From the point of view of air shower reconstruction, it is the most different point from conventional FDs that shower detector plane (SDP) can not be determined by CRAFFT which has only single pixel. In monocular analysis, once SDP is determined, shower geometries can be determined from the arrival time of the signal, and in stereo analysis, from the intersection of SDP. However, since the CRAFFT detector has a wide field of view per pixel, only the light intensity as a function of arrival time are recorded as waveform, and it is not obvious whether the shower geometry can be determined. With the CRAFFT detector, the shower geometry, energy, and $X_{\max }$ must be determined from only the waveform.

\subsection{Detector Simulation}

In order to estimate the performance of the detector, we are developing a detector simulation. The figure 4 shows the implementation of the ray-trace simulation developed using ROBAST [4]. The simulation includes the actual configuration, for example the Fresnel lens, the spatial filter, the PMT, and shielding by the frame structure. The left side of Fig. 5 shows the spot of the Fresnel lens on the screen, which has a unique shape. The middle of Fig. 5 shows the shape of the spot on the screen reproduced by the detector simulation. It reproduces well the shadow cast by the beam to compensate for the deflection of the Fresnel lens and the unique $\mathrm{x}$-shape also. The spot size at the focal plane is $44 \mathrm{~mm}$, which is small enough compared to the 5 inch PMT as shown in the right side of Fig. 5. The spot size is defined as the area that contains $95 \%$ of the incident light. The left side of Fig. 6 shows a contour map of the angular dependence of the incident light on the PMT. To reduce the computation time, this map of incident light intensity is used to simulate the

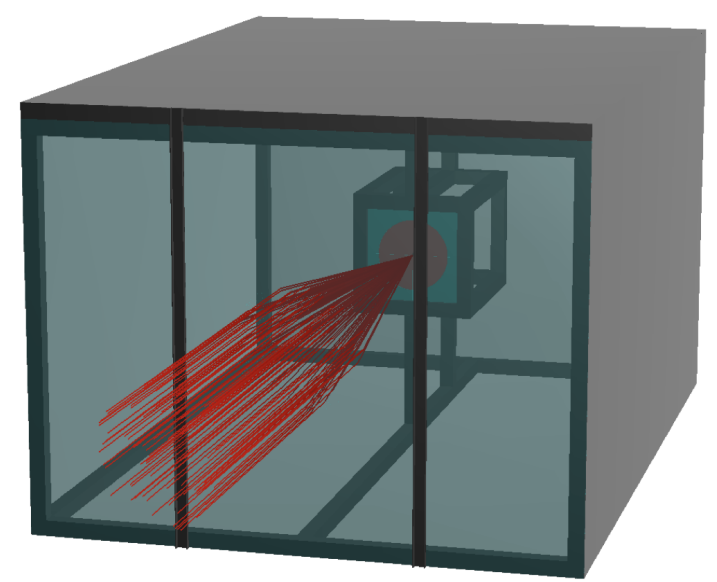

Figure 4: Raytracing simulation of CRAFFT detector with actual detector configuration. 
waveform. The right side of Fig. 6 shows the simulated waveform assuming the same conditions as the air showers obtained from the test observation as shown in Fig. 3, and the data is in good agreement. The simulated waveform uses the nightsky background data from the test observation and adds background noise. The simulations show that $10^{20} \mathrm{eV}$ air showers within $30 \mathrm{~km}$ of the detector can be triggered [5].
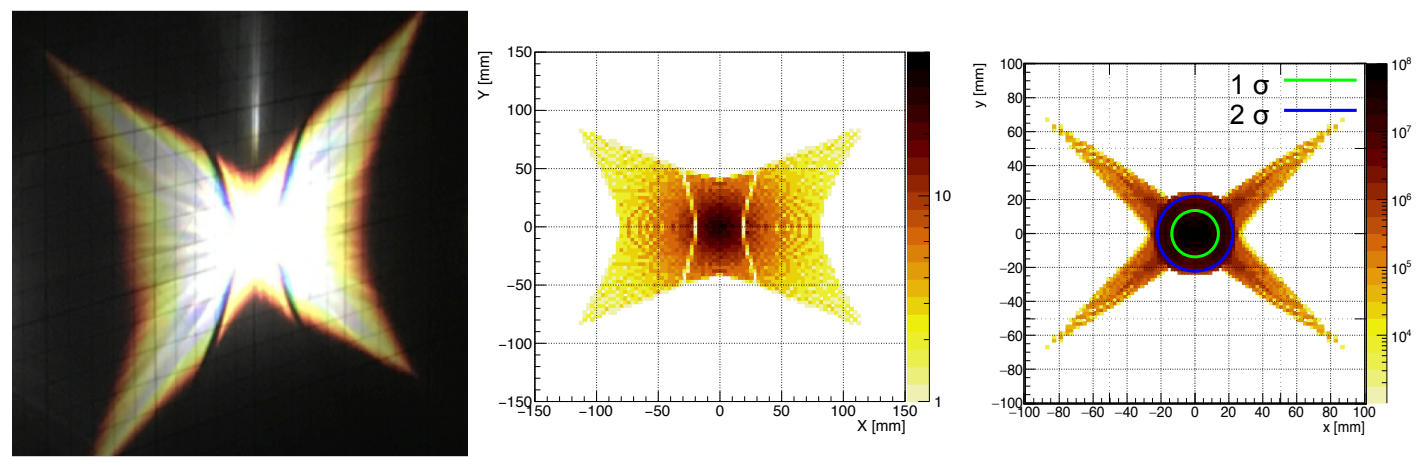

Figure 5: Actual spot shape (left) and simulated spot shape (middle) of the Fresnel lens on the screen and simulated spot size at the focal plane (right).
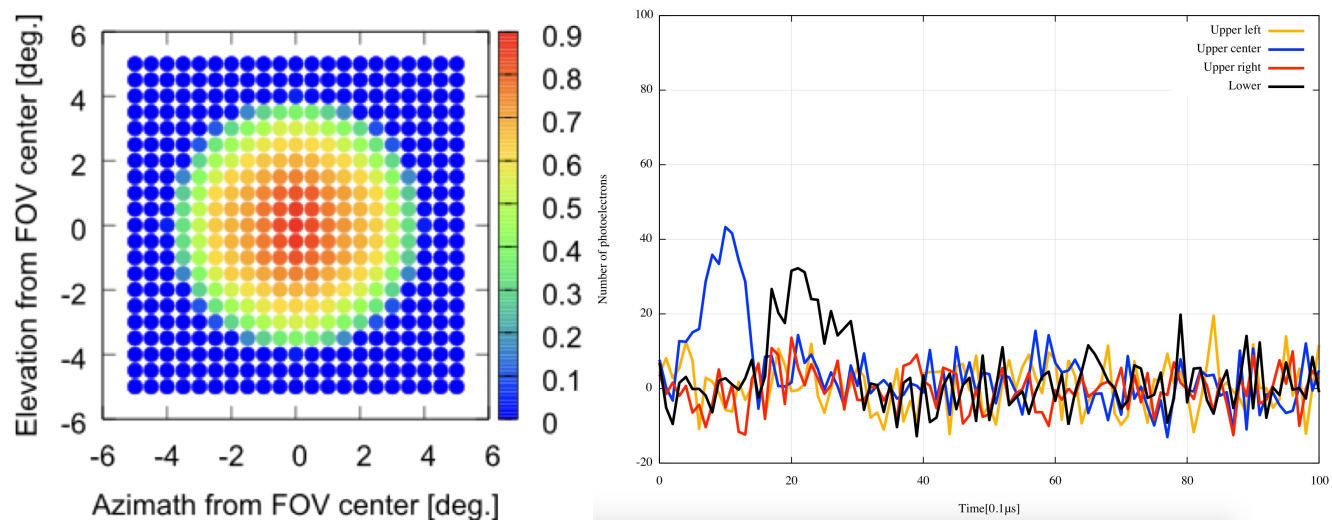

Figure 6: Angular dependence of light collective efficiency (left) and simulated waveform (right).

\subsection{Air shower reconstruction}

In the case of CRAFFT, air showers cannot be reconstructed using the same method of FDs as used in TA and Auger which can determine shower detection plane. Therefore, we have developed our own analysis method. The CRAFFT detector records waveform data with $80 \mathrm{MHz}$ FADC as shown in Fig. 3. The shape of the waveform generated by the simulation depends on the shower parameters. Therefore, when the air shower parameters of the observed waveform match the parameters of the simulation, the waveform will also match. In the waveform fitting analysis, the shower parameters are determined by searching for the minimum chi-square obtained from the simulation and data waveforms. The fit parameters should be six parameters: energy, $X_{\max }$, zenith angle, azimuth angle, and core position. In this work, energy and $X_{\max }$ were fixed, and we tried to a four-parameter fitting of the shower geometry. The figure 7 shows the results of a grid search 

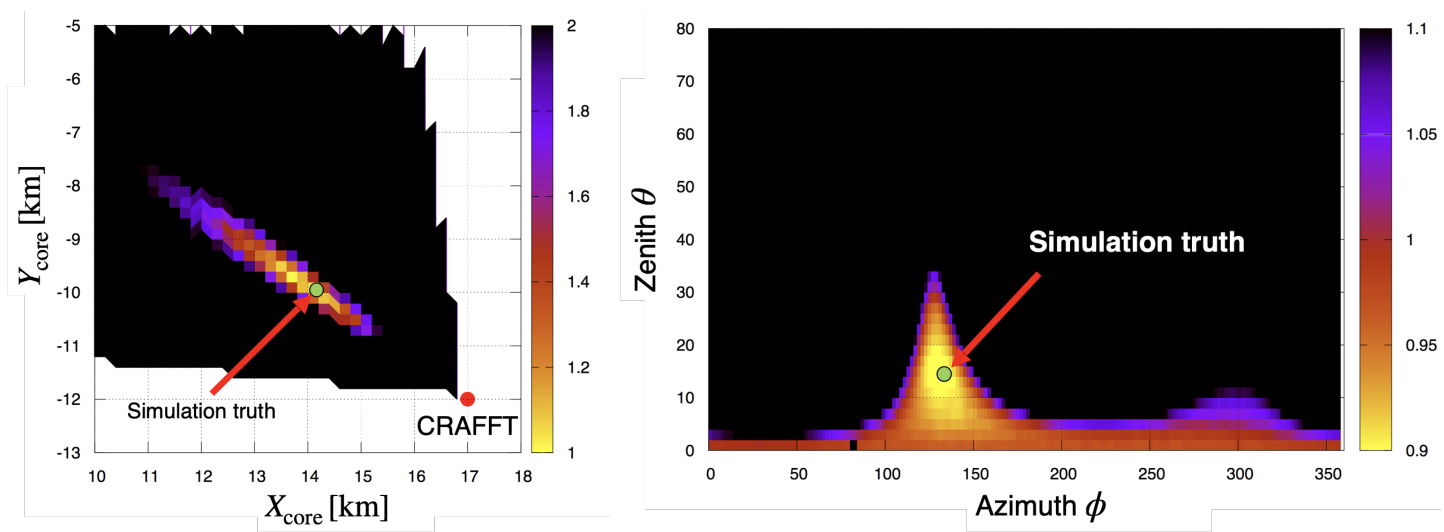

Figure 7: Distribution of reduced chi square as a function of parameters of air shower geometry in simulation study.
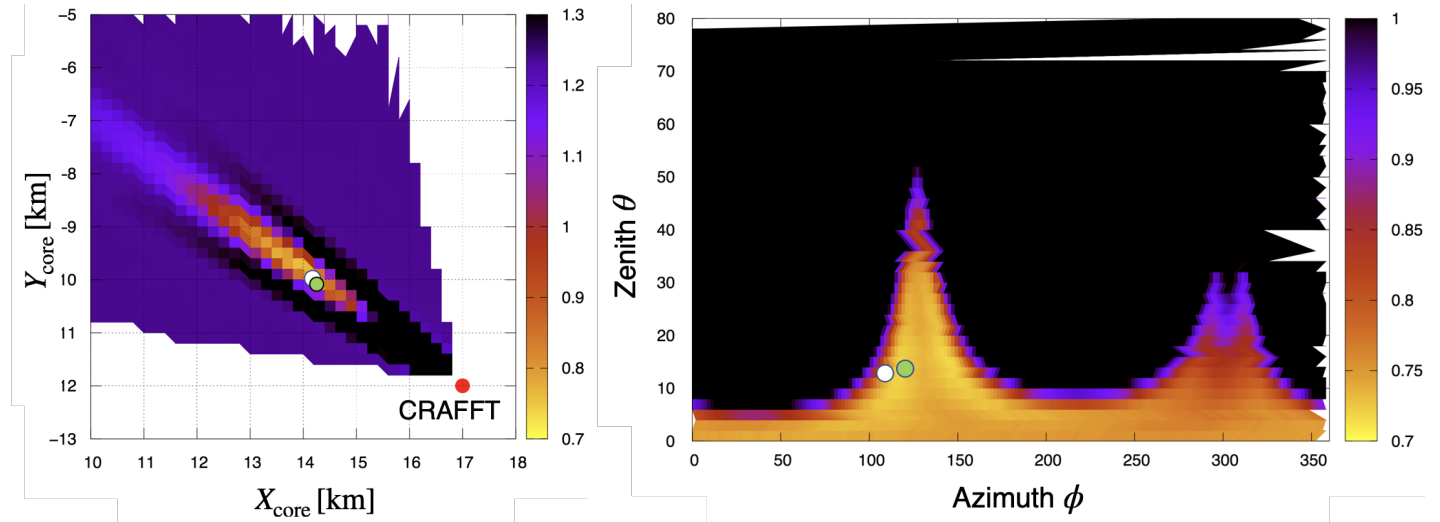

Figure 8: Distribution of reduced chi square as a function of parameters of air shower geometry applying to the observed data.

for the minimum chi-square. The chi-square converges to the simulation condition, indicating that the waveform fitting is working well. The figure 8 shows the results of applying to real data. The results of the simulation evaluation and the analysis of the real data are shown in the table 1 . In the waveform fitting using the real data, the difference in core position is $210 \mathrm{~m}$ and the opening angle is $3.3^{\circ}$. CRAFFT uses large window PMTs and is very sensitive to gain uniformity. Therefore, calibration of the PMT gain is very important. In this analysis, the non-uniformity of the PMT gain was not measured for the test observation and is not reflected.

Although CRAFFT assumes stereo observations at two or more locations, this work shows the possibility to be able to determine the shower parameters by monocular analysis as well. In the six-parameter fit, it is important to provide appropriate initial values for the convergence of the chisquare. The figure 9 shows the chi-square contour map under the condition of stereo observation. As can be seen, stereo observations are useful for determining initial values. In addition, it also can be expected that stereo observations should be able to improve the accuracy of reconstruction.

We have shown the possibility of the observation and reconstruction by the prototype detector used in the test observation. We are optimizing the detector to improve the accuracy of the 
Table 1: Comparison of the reconstructed air shower parameters of simulation study and actual data with TA FD reconstruction.

\begin{tabular}{crrr}
\hline & TA FD Reconstruction & $\begin{array}{r}\text { Result of waveform fitting } \\
\text { by simulation study }\end{array}$ & $\begin{array}{r}\text { Result of waveform fitting } \\
\text { for actual data }\end{array}$ \\
\hline \hline$\theta$ & $14.2^{\circ}$ & $18^{\circ}$ & $14^{\circ}$ \\
$\phi$ & $132.4^{\circ}$ & $128^{\circ}$ & $146^{\circ}$ \\
$X_{\text {core }}$ & $14.18 \mathrm{~km}$ & $13.8 \mathrm{~km}$ & $14.25 \mathrm{~km}$ \\
$Y_{\text {core }}$ & $-9.95 \mathrm{~km}$ & $-9.8 \mathrm{~km}$ & $-10.15 \mathrm{~km}$ \\
\hline
\end{tabular}

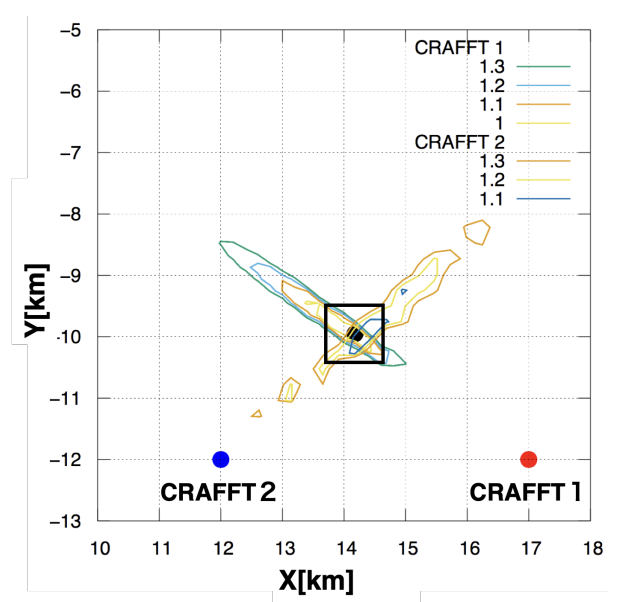

Figure 9: Contour map of reduced chi square for stereo observation.

reconstruction and to increase the field of view per telescope. For example, as shown in the figure, we are investigating a configuration with multiple smaller PMTs. We are also working on the calibration of gain uniformity, assuming a smaller PMT (Hamamatsu, R877) for optimization. For more details, please see Y. Kubota ICRC2019 [6].

\section{Future prospect and summary}

We've already confirmed the concept of detector using 1st prototype as the phase 1 . We succeeded to detect UHECR air showers. Now we are in phase 1.5 to optimize the detector configuration to improve the cost performance and the accuracy of air shower reconstruction. We are also testing our automatic operation system (T. Tomida ICRC2019 [7]) to reduce the cost of not only the detector but also the operation. For the next step, we are planning to deploy more CRAFFT detectors for stereo observation as phase 2. Our final goal is to realize a huge observatory to clarify the origins of UHECRs. If we realize the huge observatory 10 times larger than TAx4 considering $10 \%$ duty cycle. We need 500 simple FD stations which have $360^{\circ}$ viewing resultant to be $400,000 \mathrm{~km}^{2}$. It might be difficult to make such huge observatory at the same place. However, CRAFFT detector is so easy to deploy that the detector array can be distributed to several places in the world. We also think our detector concept match the plan of GCOS[8]. It is also discussed 
in the letter of intent for snowmass2021[9] that fluorescence detector array like CRAFFT or FAST [10] is one of the option for a large array for UHECR observation.

\section{Acknowledgements}

This work partially was supported by the Japan Society for the Promotion of Science (JSPS) KAKENHI Grant Numbers 25610051, JP16K17710, 21K03605, the joint research program of the Institute for Cosmic Ray Research (ICRR), The University of Tokyo, and the collaborative project of Fundamental Electronics Research Institute of Osaka Electro-Communication University. The Telescope Array Collaboration supported CRAFFT as an associated experiment.

\section{References}

[1] R. U. Abbasi et al., Astrophys. J. Lett. 790, L21 (2014).

[2] P. Privitera et al., Internal Symp. on Future Directions in UHECR Physics (2012).

[3] Y. Tameda, T. Tomida, M. Yamamoto, H. Iwakura, D. Ikeda, and K. Yamazaki, PTEP 2019 no. 4, (2019) 043F01.

[4] A. Okumura et al., Astroparticle Physics 76, 38-47 (2016).

[5] Y. Tameda et al., PoS(ICRC2019), 435 (2019).

[6] Y. Kubota et al., PoS(ICRC2021), 216 (2021).

[7] T. Tomida et al., PoS(ICRC2021), 215 (2021).

[8] R. Engel, A. Haungs, M. Roth et al., Ultra High Energy Cosmic Rays 2018 (2018).

[9] Jörg R. Hörandel et al., Snowmass2021 - Letter of Interest (2021).

[10] T. Fujii et al., Astropart. Phys. 74 (2016) 64-72. 


\section{CRAFFT Collaboration}

Yuichiro Tameda ${ }^{1}$, Takayuki Tomida ${ }^{2}$, Daisuke Ikeda ${ }^{3}$, Katsuya Yamazaki ${ }^{4}$, Takashi Kagitani ${ }^{1}$, Norimichi Shibata ${ }^{1}$, Eiji Nishio ${ }^{1}$, Hirokazu Iwakura ${ }^{2}$, Yuya Nakamura ${ }^{2}$, Yuto Kubota ${ }^{2}$, Shinnosuke Kurisu² ${ }^{2}$, and Ryosuke Saito ${ }^{2}$

${ }^{1}$ Osaka Electro-Communication University, Department of Engineering Science, Neyagawa, Osaka, Japan. ${ }^{2}$ Shinshu University, Faculty of Engineering, Nagano, Nagano, Japan. ${ }^{3}$ Kanagawa University, Faculty of Engineering, Yokohama, Kanagawa, Japan. ${ }^{4}$ Chubu university, Engineering Science Laboratory, Kasugai, Aichi, Japan. 